Original Article

\title{
Kinetic changes in the activity of HR-peroxidase induced by very low doses of phenol
}

\author{
Elzbieta Malarczyk (PhD) \\ Biochemistry Department, UMCS, Lublin, Poland
}

\begin{abstract}
The allosteric protein of horseradish peroxidase (HRP) shows two main types of activity, peroxidase and oxidase, depending on the kind of low molecular effectors. The effects of very low doses of phenol, prepared by successive dilutions in water or in $75 \%$ ethanol, on initial HRP activity in oxidation of o-dianisidine or luminol were tested in a systematic manner by colorimetric and luminometric methods. Results showed that phenol dilutions, including those below Avogadro's number, could activate or inhibit HRP in peroxidase and oxidase-type reactions with a sinusoidal pattern. Km values for the studied substrates changed parallel to HRP peroxidase/oxidase activity and the maximum activity in the peroxidase reaction corresponded to the minimum activity in the oxidase reaction and vice versa. The effect also depended on the type of dilutor. The observations of the peroxidase/oxidase oscillations in the sinusoidal pattern of HRP activity, dependent on the rate of phenol dissolution and the time of pre-incubation, point out to the conclusion that HRP might be a good model for high dilutions research. The experiments provide strong evidence that horseradish peroxidase (HRP) is a very sensitive detector of subtle changes in the concentration of phenol used as a cofactor in the peroxidaseoxidase reaction.
\end{abstract}

Keywords: HR-peroxidase, peroxidase-oxidase, phenol, hormesis, homeopathy, high dilutions

\section{Introduction}

In the cells of living organisms, the metabolism of oxygen is strictly associated to the presence of $\mathrm{H}_{2} \mathrm{O}_{2}$, a molecule that plays a crucial role in the detoxification of the environment and functions as a messenger between catabolism and anabolism [1]. This small molecule is very mobile and readily crosses biological membranes. Regulation of membrane permeability to various oxygen radicals can serve the purpose of cell protection against oxidative stress [2]. As shown by Antunes and Cadenas [3], submicromolar quantities of hydrogen peroxide are capable of inducing subtle biochemical events such as apoptotic processes. Yet, in spite of the large empirical knowledge about its crucial role in the life of cells, the molecular metabolism of $\mathrm{H}_{2} \mathrm{O}_{2}$ is still under discussion.

Among the most important destructors of hydrogen peroxide are peroxidases, exoenzymes belonging to the category of oxidoreductases, catalyzing the oxidation of different phenolic aromatics by hydrogen peroxide in the presence of oxygen [4], Peroxidases are distinguished by the kind of aromatic substrate.

In plants and fungal material, several kinds of these enzymes are recognized [5] but the most common is HR-peroxidase (horseradish peroxidase; E.C.1.11.1.7), which mainly catalyzes oxygendependent reactions of phenols and amino phenols [6]. Its red-ox potential for the pair $\mathrm{Fe}(\mathrm{III}) / \mathrm{Fe}(\mathrm{II})$ in the heme-center is the lowest among peroxidasetype enzymes, including lignin-peroxidase, cytochrome c peroxidase, and Mn-peroxidase [7]. HR-peroxidase co-operates with other enzymatic conglomerates with anti-oxidative and anti-stress properties, such as SOD, NADH oxidase, glucose oxidase, laccase, and many others which are competitive against phenolic substrates. Such multicomponent detoxification, involving biodegradation of $\mathrm{H}_{2} \mathrm{O}_{2}$ and natural phenolics, should be recognized for its practical value [8]. 
The discovery of the double catalytic role of peroxidase, described in detail by Bronnikova, Olsen and Schaffer $[9,10]$, advanced the recognition of the oscillatory character of peroxidase-oxidase (PO) reactions. According to Hauser et al. [6] and Olsen et al. [11], these reactions reveal the nature of the biological oscillator and can be modified by various metabolic co-substrates [12]. Among the activators promoting PO oscillations, some important aromatic compounds can be mentioned. Apart from phydroxybenzoate, phenol, and many natural and synthetic phenolics [13], also melatonin, which is a tryptophan-related pineal gland hormone of vertebrates, associated to circadian and seasonal rhythms, is recognized as a substrate for oneelectron reduction by HRP [14,15]. Among many substrates, guaiacol and $o$-dianisidine are the most useful in colorimetric assays of HRP activity [16] and also play a role in protecting the enzymatic active center [13]. Other authors, among them Nicell and Wright [17] and Valderrama et al., [5], underline that even a catalytic concentration of hydrogen peroxide can auto-inactivate all kinds of peroxidases and hemoproteins. There are also some other biologically active substances, e.g., ascorbic acid [12], which play a positive role in the regulation of PO oscillations by protecting the enzyme against oxidative destruction.

Taurog et al. [18] reported that small amounts of phenolics present in a reaction medium were able to change the catalytic activity of peroxidases, observable as a change in light absorption. These authors found that catechol changed the color of the reaction from amber to blue as a result of the formation of biphenoquinone when guaiacol was used as the main reactant. Doegre et al. [16] studied the mechanism of this reaction in detail and concluded that the final color in the peroxidase reaction was determined by intermediating phenoxy radicals.

Another aromatic substrate, luminol (3aminophtalhydrazide), is also useful for determining HRP activity with luminescence methods based on excitation of the radical dianionic form. Some phenolics, such as p-iodophenol, p-cumaric acid, phenol, and aniline enhance the luminescence effect in the peroxidase- $\mathrm{H}_{2} \mathrm{O}_{2}$-luminol reaction [19].

In our earlier papers, we presented results of colorimetric tests showing oscillations in peroxidase activity in the presence of extremely diluted phenolic substances in experiments with laboratorycultivated fungal material or pure enzymes [20,21]. Analyzing 19 different highly diluted phenolics, we observed that the selected aromatics generated their own distinct oscillation patterns. Among them, dilutions of phenol, vanillic and isovanillic acids, and their aldehydes produced very symmetrical sinusoidal curves with maxima and minima spaced at regular intervals, predictable from a mathematical equation [21]. These results were obtained in simple colorimetric assays with $o$ dianisidine and $\mathrm{H}_{2} \mathrm{O}_{2}$ as substrates. As a next step, it seemed interesting to examine the sensitiveness of pure HR-peroxidase to the presence of very low doses of phenol in luminometric and colorimetric assays.

\section{Materials and methods}

\section{Materials}

All assays were conducted using commercial HRperoxidase, $\mathrm{RZ}=3$ ( $\mathrm{RZ}=\mathrm{A} 403 / \mathrm{A} 275)$, purchased from SIGMA. The stock solution was prepared in $0.1 \mathrm{M}$ acetic buffer, $\mathrm{pH}$ 5.5, and stored for a few months in $-10{ }^{\circ} \mathrm{C}$. Before use, it was dissolved to the $\mathrm{RZ}=2$. High purity phenol was purchased from LobaChemie, Wien, Austria, and was redistilled before use.

\section{Dilutions of phenol and dissolvent}

Dilutions of phenol were prepared in $75 \%$ ethanol or in distilled water in 31 serial iterations, by the dilution procedure described in a previous paper [21]. The initial molar concentration of phenol was 1 $\mathrm{mol} / \mathrm{l}$, to be later dissolved at the ratio of 1:100. In a routine manner, $10 \mu \mathrm{l}$ of the starting solution were transferred to $990 \mu \mathrm{l}$ of the dissolvent and shaken vertically 10 times by hand [22,23]. The new solution (Pha 1) was dissolved in the same mode to Pha 2, which was then dissolved to Pha 3 , and so on until Pha 31. Since we expected some changes in HRP activity in the presence of the dissolvent resulting from dynamic dissolution, serial dilutions of $75 \%$ ethanol or distilled water were prepared in the same manner as the phenol dilutions. As a result, 4 lines of serial dilutions were obtained: 2 for phenol, marked as Pha (phenol in alcohol) and $\mathbf{P h w}$ (phenol in water), and 2 lines for the dissolvent, 75\% alcohol (A) and distilled water (W), all prepared in the same laboratory conditions.

\section{Preincubation of HRP with diluted phenol, ethanol, and water}

Incubation procedures were conducted in the same conditions for all the lines of serial dilutions. 2 milliliters of HRP solution were mixed with $200 \mathrm{\mu l}$ of an appropriate dilution and colorimetric or luminometric assays were conducted after 4 to 24 hours according to the experimental presumption.

\section{Measurement of HRP activity}

\section{Spectrophotometric assay}

The colorimetric method described in paper [24] with $o$-dianisidine as proton donor was used in the same manner as in papers [20,21]. Measurements on a Varian Cary 2 spectrophotometer were made at $460 \mathrm{~nm}$ in $25^{\circ} \mathrm{C}$. The reaction mixture was composed of $0.2 \mathrm{ml}$ of the sample, $2.8 \mathrm{ml}$ of acetic buffer $(\mathrm{pH}$ 
5.5), $3 \mathrm{mmol}$ of $\mathrm{H}_{2} \mathrm{O}_{2}$, and $12 \mathrm{mmol}$ of $o$-dianisidine. The activity was expressed in nkatal/l calculated as absorbance value with factor 1470 .

\section{Luminometric assay}

The reaction mixture consisted of $0.5 \mathrm{ml}$ of the HRP solution (after incubation with an appropriate phenol dilution), $0.5 \mathrm{ml}$ of distilled $\mathrm{H}_{2} \mathrm{O}, 100 \mu \mathrm{l}$ of luminol $(1.5 \mathrm{mmol})$, and $50 \mu \mathrm{l}$ of $\mathrm{H}_{2} \mathrm{O}_{2}(3 \mathrm{mmol})$. The temperature of the experiments was $25^{\circ} \mathrm{C}$. The assay was performed immediately after the addition of hydrogen peroxide, and chemiluminescence was measured after 10 seconds in a Lumat LB 9507 luminometer (Berthold Technologies). The results were calculated from 3 repetitions. Because the standard deviation (SD) value usually did not exceed 200 RLU (Relative Light Units), it is not shown in the figures. To adjust the sinusoidal shape of the experimental curves, a recalculation using a 4-parameter equation $\mathrm{y}=\mathrm{y}_{0}+\mathrm{A} \cos (B n-\mathrm{y})($ when $n$ is number of iteration) with Levenberg-Marquardt algorithm in the method of least squares [25] was performed as previously described [21].

\section{Kinetics and Km determination}

Kinetic dependencies for HRP were determined after 4-hour incubation with 6 phenol dilutions selected from the Pha series in a colorimetric assay with growing concentrations of $o$-dianisidine and $\mathrm{H}_{2} \mathrm{O}_{2}$. The results were expressed in $\mu \mathrm{mol}$ and the $\mathrm{Km}$ values were calculated from Michaelis-Menten plots and also double reciprocal plot according to Lineweaver-Burk were evaluated where [S] means substrate concentration and $\mathrm{V}$ applies to velocity of reaction.

\section{Results}

I. Changes in HRP activity in the presence of low doses of phenol diluted in ethanol medium

Activity of HRP pre-incubated with 2 parallel series of phenol dilutions prepared in $75 \%$ ethanol and marked as Pha line was measured by luminometric assay. A compilation of all the results yielded a sinusoidal curve with 3 distinct activity maxima and minima (Figure 1).

It is important to note that the mentioned sinusoidal changes in HRP activity were induced after 4-hour pre-incubation of HRP protein with very low doses of phenol added before contact of the enzyme with an appropriate substrate (luminol in the luminometric assay or $o$-dianisidine in the colorimetric method), which showed the same sinusoidal shape [21]. The marked amplifications or reductions in activity took place at regular distances between the phenol dilutions equal to 10 centesimal dilutions. Based on the experimental data, the following dilutions were selected for further experiments: Pha 1, 11, 21, and 31 as dilutions lowering (inhibiting) HRP activity and Pha 6, 16, and 26 as dilutions enhancing (activating) HRP activity.

Figure 1: Changes in HRP activity after 4-hour incubation with 2 independent series of phenol dilutions in $75 \%$ ethanol (Pha). Luminometric assay; the control value was $17 \times 10^{3} \mathrm{RLU}$; the dashed line corresponds to the graphic expression of a theoretical equation (see Materials and Methods).

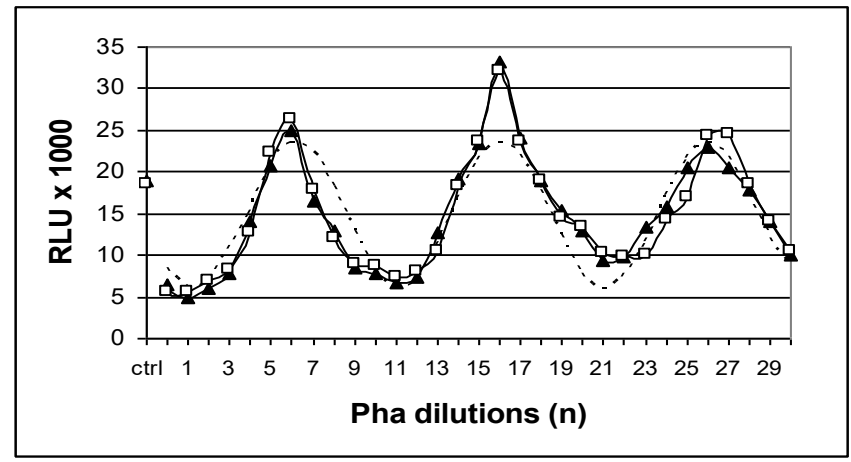

Numerical fitting of the proposed 4-parameter equation with experimental data allows to determine the coefficients as: $\mathrm{y}_{0}=15 \times 10^{3} ; \mathrm{A}=9,0 \times 10^{3}$; $B=2 \Pi / 10 ; \mathrm{y}=3,8$. The coefficient yo can be compared to the control value $17 \times 10^{3}$ [RLU], while the amplitude A represents the oscillatory effect induced by Pha dilutions. B-value describes the 10 dilution period, and $\mathrm{Y}$ is the phase shift probably due to some ponderal effect of phenol, but not well understood yet, as well the minimum-value activity $\left(5 \times 10^{3} \mathrm{RLU}\right)$, probably due to some solvent property, related to its own dynamics or some gas dissolved during dilution.

\section{The effect of dilutor type on the changes in HRP activity}

Subsequent luminometric experiments showed clear differences in the action of the 7 chosen dilutions of phenol dependent on the kind of dilutor. It ought to be underlined that after 4-hour incubation, the phenol-water line (Phw) produced smaller effects than the phenol-ethanol line (Pha), and the values of the latter series increased after 24 hours. The effect of diluted distilled water was very small and faded after 24 hours. The $75 \%$ ethanol line, on the other hand, showed a tendency to increase its values after 24 hours. A comparison of all line activities after 4 and 24 hours is presented in Fig. 2. The results confirm the appropriateness of choosing $75 \%$ ethanol for the preparation of phenol dilutions enabling stabilization of the studied effect.

\section{Time-dependent effect of phenol-ethanol series on HRP activity}


Changes in HRP activity during 12-hour preincubation with the selected maximal and minimal phenol-ethanol dilutions were examined using luminometric test. The activity was measured every 2 hours without adding new portions of the diluted phenol. Two maxima in the activity after 4 and 12 hours of incubation and one minimum after 8 hours were observed for dilutions Pha 6, 16, and 26 (Figure 3). The lowest activity was observed for dilutions Pha 1, 11, 21, and 31 which placed lower than the control. The diagrams showed several dynamics in HRP activity, which were dependent on the rate of phenol dissolution and the duration of pre-incubation.

Figure. 2: Comparison among 4 variants of HRP incubation after 4 and 24 hours with chosen dilutions: A - series of $75 \%$ ethanol, Pha - series of phenol in $75 \%$ ethanol, W - series of distilled water, Phw - series of phenol in water. Luminometric assay.

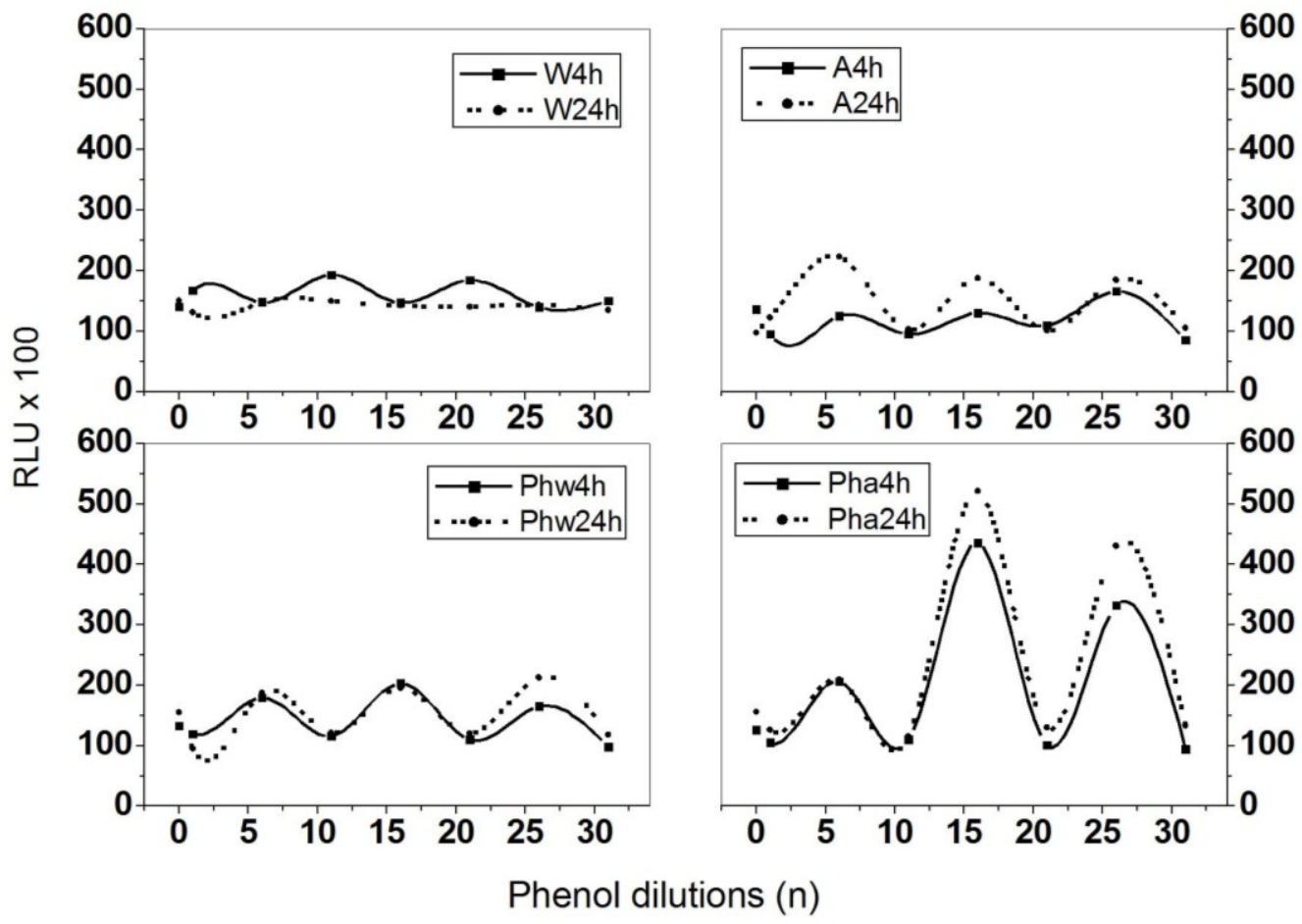

\section{Km values}

For individual dilutions selected from the Pha line, the Michaelis-Menten kinetic relations based on colorimetric assay against the various concentrations of $\mathrm{o}$-dianisidine as proton donor and $\mathrm{H}_{2} \mathrm{O}_{2}$ were determined. For comparison, 3 kinetic graphs for Pha 6, Pha 21, and control were chosen and they are shown in Figure 4.

These 3 lines were calculated from data of hyperbolic Michaelis-Menten plot according to Lineweaver-Burk reciprocal plot and Pha 6 line, placed under control line, show the enzymatic activation of this dilution by comparison to Pha 21 line, which corresponds to inhibition of HRP activity.

$\mathrm{Km}$ values were calculated for all tested kinetic graphs (Fig. 5). Lower Km values were observed for the "positive", activating dilutions, Pha 6, 16, and
26, as opposed to the "negative" or inhibiting dilutions Pha 11, 21, and 31.

\section{Effect of hydrogen peroxide on HRP activity}

During luminescence measurement, 2 parallel series of assays were performed to determine the chemiluminescence of a same sample with or without $\mathrm{H}_{2} \mathrm{O}_{2}$. $\mathrm{HRP}$ incubated with Pha 6, 16 and 26 showed maximal peroxidase activity and minimal oxidase activity, whereas HRP incubated with Pha 1, 11 and 21 gave opposite results (Figure 6).

Figure 3: Time relations among 7 chosen dilutions of Pha, incubated with HRP during 12 hours. Luminometric assay. 


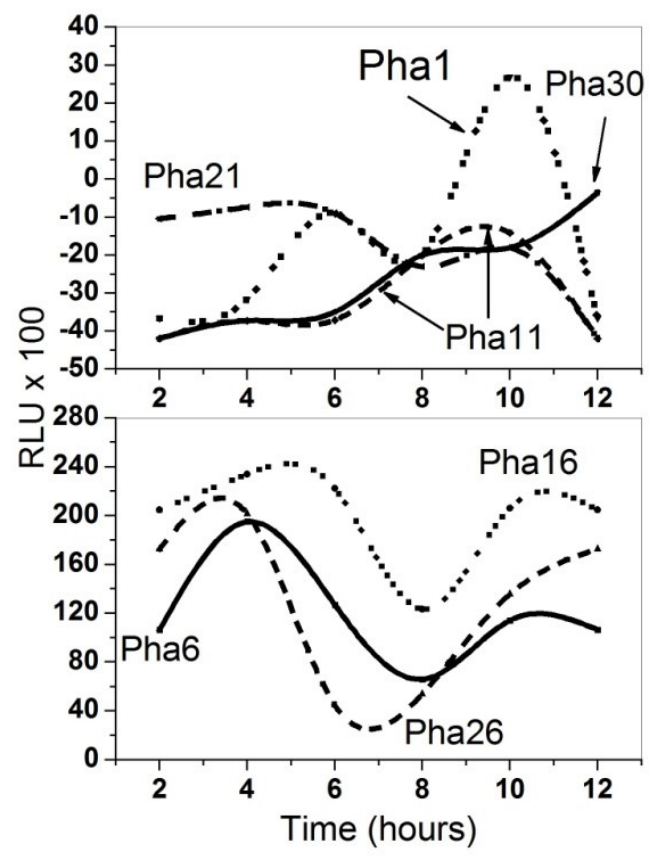

Figure 4: Lineweaver-Burk plot for HR-peroxidase incubated with phenol dilutions for control (75\% ethanol) squares, Pha 6 (HRP activation) circles, and Pha 21 (HRP inhibition) triangles; V - velocity of reaction in nkatals/L, [S] - substrate concentration ( $\mu \mathrm{mols}$ of $o$-dianisidine). Colorimetric assay.

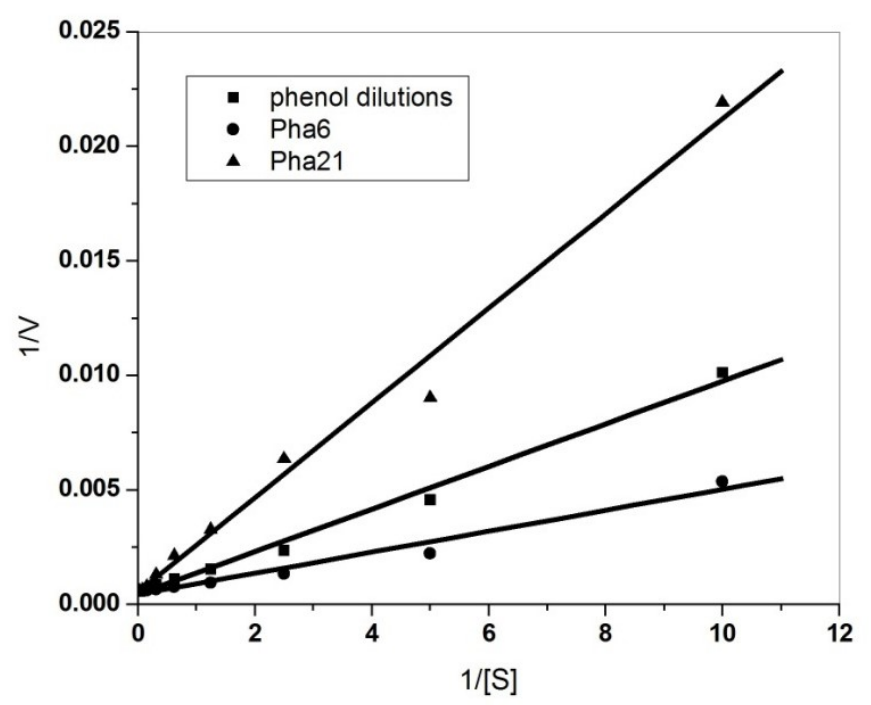

Figure 5: Km values ( $\mu$ mols) for HRP incubated for 4 hours with selected dilutions from the Pha series; Ctrl - control with $75 \%$ ethanol. Colorimetric assay.

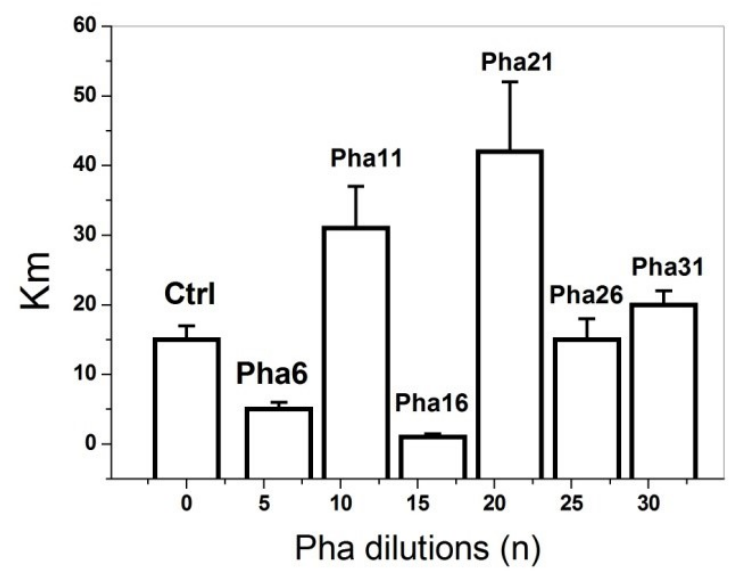

Figure 6: The effect of hydrogen peroxide $(3 \mathrm{mM})$ on results of luminometric assay of HRP activity after 4 hour-incubation with appropriate dilutions from the Pha series; white squares - luminol alone; black squares - luminol and $\mathrm{H}_{2} \mathrm{O}_{2}$.

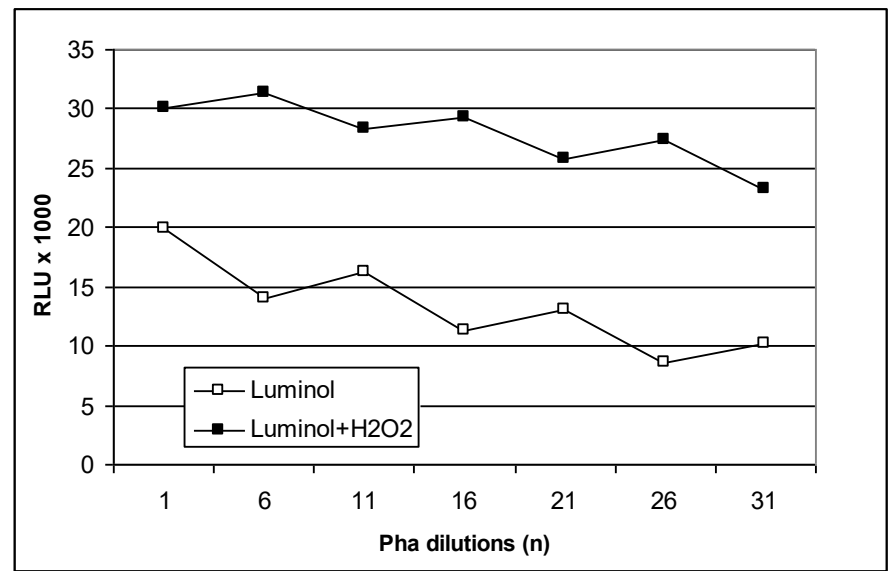

\section{Discussion}

During incubation of HRP solution in the presence of high dilutions of phenol in water or in $75 \%$ ethanol, marked changes in the activity of the enzyme were observed. The sinusoidal shape of these results revealed regular changes in enzyme activity, oscillating above or below the control for the selected dilutions.

The choice of HRP, an enzyme associated with two substrates, one acting as proton donor and the other, $\mathrm{H}_{2} \mathrm{O}_{2}$, playing an important role in red-ox variations of heme in the active center of the enzyme, is not accidental, as it allows to exploit two kinds of activity known as peroxidase-oxidase reaction [11]. Phenolics, simple phenol among them, are recognized as natural agents inducing oscillations in the peroxidase-oxidase reaction [6]. Phenol belongs to the category of peroxidase co- 
substrates which increase the luminescence of system luminol- $\mathrm{H}_{2} \mathrm{O}_{2}$-HRP [19]. In addition, phenol is produced in the natural environment as a result of biodegradation of natural aromatics, and is common in areas devastated by industry.

The key factor in the modification of peroxidase activity by very low doses of phenol seems to be the activation of the latter through shaking during the procedure of dilution [26]. When the dilutions were pre-incubated with the enzyme, a regular sinusoidal curve was obtained instead of the anticipated gradual linear disappearance of phenol concentration. Theoretically, no changes in the activity of HRP should be observed in dilutions of phenol over Avogadro's number. However, the regularity of the extreme minimum or maximum points on the sinusoid curve and the repeatability of this phenomenon may challenge our current knowledge about the response of enzymes to very low doses of co-factors. In several papers, Elia and co-workers [22,23] proved that there were distinct changes in calorimetric assays of highly diluted acids, bases, and salts which were activated by manual or mechanical shaking during the process of dilution by comparison to their moderate dilutions. On the other hand, no sinusoidal, oscillating dynamics was observed during those experiments, which seems to be reserved for biological models only where the semiconductor properties of proteins and other natural biopolymers can serve as detectors of fine changes in molecular kinetics.

Oscillating changes in HRP activity can be interpreted in agreement with the phenomenon of hormesis as positive or negative states of kinetic overcompensation, according to Calabrese's proposition [27,28]. The regular pattern of changes observed by us in HRP activity leads us to believe that dilutions of phenol seemed to influence the protein structures in the catalytic center of HRP, causing unstable disorders in their molecular connections, visible as periodic changes in the interaction between the enzyme and the substrate.

In a previous paper we observed that various phenolics induced different shapes of sinusoidal curve, and HRP, when incubated with them, could detect those differences [21]. This was observed for 19 phenolic co-substrates of peroxidase. Those observations are in agreement with the fact that the particle may have different molecular composition and provide a basis for discriminating the various molecules according to their characteristic frequency in resonance techniques.

The wave of dynamic dissolutions for phenol, detected by HRP, was very similar in shape and frequency to vanillates and isovanillates, each possessing one $\mathrm{OH}$ group. Description of several waves of dissolution for various effectors may become a new analytical instrument in studies of the kinetics of enzymes co-working with them. In our experiments, the frequency of the phenol oscillation frequency (Fig 1), detected by HRP, revealed a period of 10 dilution steps.

Nearly the same oscillation effect and values of period between maximum and minimum points on a centesimal dilution curve were described in an article by Belon [29] in connection with basophile degranulation inhibited by progressive dilutions of histamine. Many other studies of bimodal enzymatic response to various effectors dilutions are mentioned in a review by Witt et al. [30], among them several papers by Dittmann and Harisch [3133]. However, the effect of sinusoidal changes of enzyme activity in response to a systematic progression in dilution of the various tested effectors was not discussed in these papers. It ought to be stressed that the changes in HRP activity discussed in this study were highly repetitive and measurable with spectral and luminometric methods.

In nature, the peroxidase molecule plays the role of natural biological oscillator [11], where the enzyme activity oscillates between two extreme points on a sinusoidal curve. This was also visible in the presented experiments, when the peroxidase was incubated with successive phenol dilutions. During these sinusoidal changes, the highest kinetic energy seems to correspond to the highest peroxidase activity and the highest potential energy is connected mainly with the extreme oxidase activity.

Olsen et al. [11] suggested that peroxidase protection against damage by oxygen corresponds to the level of superoxide radicals. It is possible that the dynamic dilutions of phenol (co-substrate) keep these free radicals at balance, which facilitates protection of the active center of HRP $[13,17]$ by the phenolic substrate (luminal, o-dianisidine). The results of our experiments with $\mathrm{Pha}$ series prove that ethanol used as a dilutor can stabilize this balance better than water.

In spite of the lack of full understanding of the described occurrences, and of the phenomenon of hormesis in general, the unique properties of HRperoxidase and other oxizymes [20,21,34] as detectors of ultra low doses of their co-substrates should be recognized.

The present results are compatible with those of other authors who studied similar biological phenomena where enzymes play a crucial role [30]. The "waves" of dynamic dilutions of effectors measured as changes in the enzymatic activity of HRP have a repetitive nature and are measurable with physicochemical methods such as spectrophotometry, luminometry or electrophoresis (paper in preparation). In the future, the study of enzymatic kinetics may be broadened to include a new branch of knowledge connected with low and very low doses of substrates and effectors. This new 
science could find its application in planning plant preservation due to the possibility of considerably decreasing the use of toxic chemical ingredients $[35,36]$.

\section{Acknowledgements}

The author thanks dr Janina Rodakiewicz-Nowak, $\mathrm{PhD}$, for her valuable remarks and stimulating discussion and Janina Kochmańska-Rdest, MSc, for her excellent technical assistance. The work was supported by Polish Scientific Project BW/UMCS 2007.

\section{References}

[1] Rhee SG, Chang TS, Bae YS, Lee SR, Kang SW. 2003. Cellular regulation by hydrogen peroxide. J Am Soc Nephrol. 2003; 14: S211-S215.

[2] Sousa-Lopes A, Antunes L, Cyrne L, Marinho HS. Decreased cellular permeability to $\mathrm{H}_{2} \mathrm{O}_{2}$ protects Saccharomyces cerevisiae cells in stationary phase against oxidative stress. FEBS Lett. 2004; 578: 152-156.

[3] Antunes F, Cadenas E. Cellular titration of apoptosis with steady state concentrations of $\mathrm{H}_{2} \mathrm{O}_{2}$ : submicromolar levels of $\mathrm{H}_{2} \mathrm{O}_{2}$ induce apoptosis through Fenton chemistry independent of the cellular thiol state. Free Radical Biol Med. 2001; 30(9): 1008-1018.

[4] Halliwell B, Clement MV, Ramalingam J, Long LH. Hydrogen peroxide. Ubiquitous in cell culture and in vivo? IUBMB Life. 2000; 50(4): 251-257.

[5] Valderrama B, Ayala M, Vazquez-Duhalt R. Suicide inactivation of peroxidases and the challenge of engineering more robust enzymes. Chem Biol. 2002; 9: 555-565.

[6] Hauser MJB, Olsen LF. The role of naturally occurring phenols in inducing oscillations in the peroxidase-oxidase reaction. Biochemistry. 1998; 37: 2458-2469.

[7] Banci L, Bertini I, Turano P, Tien M, Kirk TK. Proton NMR investigation into the basis for the relatively high redox potential of lignin peroxidase. Proc Natl Acad Sci. 1991; 88: 6956-6960.

[8] Nakayama T, Amachi T. Fungal peroxidase; its structure, function, and application. J Mol Catal B: Enzymatic. 1999; 6: 185-198.

[9] Bronnikova TV, Schaffer WM, Olsen LF. Nonlinear dynamics of the peroxidase-oxidase reaction: I. Bistability and bursting at low enzyme concentration. J Phys Chem B. 2001; 105: 310-321.

[10] Schaffer WM, Bronnikova TV, Olsen LF. Nonlinear dynamics of the peroxidase-oxidase reaction: II. Compatibility of an extended mechanistic model with previously reported modeldata correspondences. J Phys Chem B. 2001; 105: 5331-5340.

[11] Olsen LF, Hauser MJB, Kummer U. Mechanism of protection of peroxidase activity by oscillatory dynamics. Eur J Biochem. 2003; 270: 2796-2804.

[12] Gan N, Cai R, Lin Zh. Determination of ascorbic acid based on a peroxidase oscillator reaction. Anal Chem Acta. 2002; 466: 257-260.

[13] Adak S, Mazumder A, Banerjee RK. Probing the active site residues in aromatic donor oxidation in horseradish peroxidase: involvement of an arginine and tyrosine residue in aromatic donor binding. Biochem J. 1996; 314: 985-991.

[14] Olsen LF, Lunding A, Lauritsen FR, Allegra M. Melatonin activates the peroxidase-oxidase reaction and promotes oscillations. Biochem Biophys Res Commun. 2001; 284: 1071-1076.

[15] Ximenes VF, Catalani LH, Campa A. Oxidation of melatonin and tryptophan by an HRP cycle involving compound III. Biochem Biophys Res Commun. 2001; 287: 130-134.

[16] Doegre DR, Divi RL, Churchwell MI. Identification of the colored guaiacol oxidation product produced by peroxidases. Anal Biochem. 1997; 250: 10-17.

[17] Nicell JA, Wright H. A model of peroxidase activity with inhibition by hydrogen peroxide. Enzyme Microbiol Technol. 1997; 21: 302-310.

[18] Taurog A, Dorris ML, Guziec FS. An unexpected side reaction in the guaiacol assay for peroxidase. Anal Biochem. 1992; 205: 271-277.

[19] Navas Diaz A, Garcia Sanchez F, Gonzales Garcia JA. Hydrogen peroxide assay by using enhanced chemiluminescence of the luminol- $\mathrm{H}_{2} \mathrm{O}_{2}$ horseradish peroxidase system: Comparative studies. Anal Chim Acta. 1996; 327: 161-165.

[20] Malarczyk E, Jarosz-Wilkołazka A, Kochmańska-Rdest J. Effects of low doses of guaiacol and ethanol on enzymatic activity of fungal cultures. Nonlinearity Biol Toxicol Med. 2003; 1(2): 176-184.

[21] Malarczyk E, Kochmańska-Rdest J, PaździochCzochra M. Effect of low and very low doses of simple phenolics on plant peroxidase activity. Nonlinearity Biol Toxicol Med. 2004; 2(2): 129-141.

[22] Elia V, Niccoli M. Thermodynamics of extremely diluted aqueous solutions. Ann N Y Acad Sci. 1999; 827: 241-248.

[23] Elia V, Elia L, Cacace P, Napoli E, Niccoli M, Savarese F. The "extremely diluted solutions" as multi-variable systems: a study of calorimetric and conductometric behavior as a function of the 
parameter time. J Thermal Analysis and Calorimetry. 2006; 84(2): 317-323.

[24] Clairborne AC, Fridovich I. Chemical and enzymatic intermediates in the peroxidation of $\mathrm{o}$ dianisidine by horseradish peroxidase. Biochemistry. 1979; 18: 2324-2330.

[25] Gill PR, Murray M, Wright MH. The Levenberg-Marquardt method. In: Practical Optimization. London: Academic Press; 1981. 136137.

[26] Zacharias CR. Physical research in dynamized systems. Med Hypotheses. 2002; 58(6): 523-526.

[27] Calabrese EJ. Overcompensation stimulation: A mechanism for hormetic effects. Crit Rev Toxicol. 2001; 31: 425-470.

[28] Calabrese EJ, Baldwin LA. U-shaped doseresponses in biology, toxicology and public health. Ann Rev Pub Health. 2001; 22: 15-23.

[29] Belon P. The effect of histamine dilutions on basophile degranulation. In: Research in Homeopathy. $4^{\text {th }}$ ed. [s.1.]: Boiron; 2006. 312-319.

[30] Witt CM, Bluth M, Albrecht H, Weißhuhn TER, Baumgartner S, Willich SN. The in vitro evidence for an effect of high homeopathic potencies: A systematic review of the literature. Compl Ther Med. 2007; 15: 128-138.
[31] Dittmann J, Kanapin H, Harisch G. Einfluss ausgewählter Homöopathika auf die katalytische Aktivität der Urikase, der sauren Phosphatase und der zytosolischen Glutation-S-Transferasen. Biol Med. 2000; 29(3): 125-131.

[32] Dittmann J, Kanapin H, Harisch G. Einfluss ausgewählter Homöopathika auf die katalytische Aktivität der cAMP-abhängigen Proteinkinase. Biol Med. 2000; 29(6): 289-294.

[33] Harisch G, Dittmann J. Unterschidlicher Einfluß von cAMP-Potenzen und cAMPVerdünnungen am Beispiel verschiedener Enzymsysteme. Biol Med. 1998; 27(2): 55-62.

[34] Malarczyk E, Paździoch-Czochra M. Multiple respiratory bursts as a response to veratrate stress in Rhodococcus cells. Cell Biol Intern. 2000; 24 (8): 515-527.

[35] Manninger K, Csõsz M, Tyihak E. Induction of resistance of wheat plants to pathogens by pretreatment with N-methylated substances. Acta Biol Hung. 1998; 49: 275-281.

[36] Tyihak E, Steiner U, Schönbeck F. Time- and dose-dependent double immune response of plants to pathogens. Proceedings of $13^{\text {th }}$ International Reinhardsbrunn Symposium. 2001 May 14-18; Friedrichroda, Germany. Friedrichroda: [s.n.]. 187196.

\section{(cc) BY-No-ND Licensed to GIRI}

Supported by Polish Scientific Project BW/UMCS 2007.

Conflict of interest: author declares there is no conflict of interest

Received: 26 December 2007; Revised 21 June 2008; Published: 30 June 2008

Erratum: 30 Dec 2008. (http://www.feg.unesp.br/ ojs/zacha_ijhdr/erratum/?v=7\&i=23\&pi=48)

Correspondence author: Elzbieta Malarczyk, malar@poczta.umcs.lublin.pl

How to cite this article: Malarczyk E. Kinetic changes in the activity of HR-peroxidase induced by very low doses of phenol. Int $J$ High Dilution Res [online]. 2008 [cited YYYY Mmm DD]; 7(23): 48-55. Available from: http://www.feg.unesp.br/ ojs/index.php/ijhdr/article/view/37/349. 